\title{
Multi-Instantons Localized at the Origin
}

\author{
Yves Brihaye \\ Physique Théorique, University of Mons, B-7000 Mons, Belgium
}

\begin{abstract}
We obtain a family of self-dual Yang-Mills fields in an SU(2) gauge theory. Some of them describe pseudoparticles with arbitrary topological numbers and with action densities concentrated around the origin.
\end{abstract}

\section{Introduction}

By now, much is known about self-duality equations (SDE) for SU(2) gauge theories. In principle, Atiyah et al. [1] have solved the problem completely, but only a restricted number of solutions are explicitly known and understood as solitons by knowledge of their action or energy density. The most popular one is the $k$-instanton discovered by' 'tHooft [2]. It corresponds essentially to a superposition of $k$ widely separated instantons [3].

Recently, research for multi-monopoles has led to new (time independent) solutions [4]; these have a cylindric symmetry, finite energy and their energy density is maximal on a circle in such a way that most of the energy is concentrated in a torus-like region of space.

In this paper, we exhibit a class of time dependent solutions with finite action and an action density maximal on a circle in Euclidean space-time. To obtain such solutions, we require a particular transformation law of the fields under the subgroup $\mathrm{SO}(2) \times \mathrm{SO}(2)$ of rotations in the $x_{1}, x_{2}$ plane and in the $x_{0}, x_{3}$ plane. The solutions belong to a large class obtained in [8] with different motivations. In Sect. 2 of the paper, we rapidly explain the ansatz and the construction; then we study in Sect. 3 the physically relevant solutions. Some conclusions are drawn in Sect. 4.

Let us first write the equations to be satisfied; in order to study the SDE

$$
F_{\mu \nu}=\tilde{F}_{\mu \nu} \equiv \frac{1}{2} \varepsilon_{\mu \nu \varrho \sigma} F^{\varrho \sigma}
$$

for the gauge field $F_{\mu v}$ defined as usual in terms of a gauge potential $A_{\mu}$ by $F_{\mu \nu}$ $=\partial_{\mu} A_{v}-\partial_{v} A_{\mu}+i\left[A_{\mu}, A_{v}\right]$, we have used the Yang formalism. It works with the light-like coordinates

$$
Y=\frac{1}{\sqrt{2}}\left(x^{0}-i x^{3}\right), \quad Z=\frac{1}{\sqrt{2}}\left(x^{2}+i x^{1}\right), \quad \bar{Y}=Y^{*}, \quad \bar{Z}=Z^{*}
$$


and, in the so called triangular or Yang gauge, the $\operatorname{SDE}(1,1)$ reduce to the Yang equations below (see [6] for more details)

$$
\begin{aligned}
\left(\frac{f_{, Y}}{f}\right)_{, \bar{Y}}+\left(\frac{f_{, Z}}{f}\right)_{, \bar{Z}}-\frac{e_{, Y} \cdot g_{, \bar{Y}}}{f^{2}}-\frac{e_{, Z} \cdot g, \bar{Z}}{f^{2}}=0, \\
\left(\frac{e_{, Y}}{f^{2}}\right)_{, \bar{Y}}+\left(\frac{e_{, Z}}{f^{2}}\right)_{, \bar{Z}}=0 \\
\left(\frac{g_{, \bar{Y}}}{f^{2}}\right)_{, Y}+\left(\frac{g_{, \bar{Z}}}{f^{2}}\right)_{, Z}=0 .
\end{aligned}
$$

The functions $f, e, g$ are the basic objects; one can show that:

(i) if

$$
f=f^{*}, \quad e=-g^{*} \quad\left(\text { respectively } e=g^{*}\right)
$$

the associated gauge quantities are in the algebra of SU(2) [respectively SU(1,1)],

(ii) the new functions defined by

$$
\begin{aligned}
& \left(f^{\prime}, e^{\prime}, g^{\prime}\right)=\frac{1}{\Delta}(f,-g,-e), \quad \Delta=f^{2}-e g, \\
& \left(f^{\prime}, e^{\prime}, g^{\prime}\right)=\left(k k^{*} f,\left(k^{*}\right)^{2} e,(k)^{2} g\right)
\end{aligned}
$$

still satisfy (1.3) and (1.4) as long as $(f, e, g)$ satisfy them [7].

\section{Construction of Solutions}

In order to simplify the problem of solving the Yang equations (1.3) and (1.4), we have found it convenient to look for sequences of solutions of the following form

$$
\begin{aligned}
S_{n} & \equiv\left(f_{n}, e_{n}, g_{n}\right) \quad n \in \mathbb{Z} \\
& =\frac{1}{K_{n-1}}\left(K_{n}, Y^{a^{n}} Z^{b^{n}}, \mu_{n} \bar{Y}^{a^{n}} \bar{Z}^{b^{n}}\right),
\end{aligned}
$$

where the $K_{n}$ 's are real functions of $r(=Y \bar{Y})$ and $s(=Z \bar{Z})$ only, $\mu_{n}$ are signs and $a_{n}, b_{n}$ are chosen to be integers throughout the paper. The ansatz (2.1) is such that, under any rotations of $\mathrm{SO}(2) \times \mathrm{SO}(2)$ with angles $(\omega, \varphi)$, each triplet $S_{n}$ transforms like a dilatation (1.7) with $k=\exp \frac{i}{2}\left(a_{n} \omega+b_{n} \varphi\right)$.

By substitution of the ansatz (2.1) into Eqs. (1.4) one can easily show that they are satisfied provided two successive $K_{n}$ are related by

$$
\begin{aligned}
& \partial_{s} K_{n}=a_{n} K_{n-1}-r \partial_{r} K_{n-1}, \\
& \partial_{r} K_{n}=-b_{n} K_{n-1}+s \partial_{s} K_{n-1} .
\end{aligned}
$$

Equations (2.2) are compatible with each other iff

$$
\left[r \partial_{r r}+s \partial_{s s}+\left(1-a_{n}\right) \partial_{r}+\left(1-b_{n}\right) \partial_{s}\right] K_{n-1}=0
$$


After elementary algebraic manipulations, one can show that the infinite system (2.2) and (2.3) is self-consistent iff $a_{n+1}=a_{n}+1$ and $b_{n+1}=b_{n}+1$. Without losing generality, we will chose in the following

$$
a_{n}=n-1, \quad b_{n}=b+n-1 \quad(b \geqq 0) .
$$

Then choosing for $K_{0}$ the most general analytic function obeying (2.3) (see appendix) we have

$$
K_{0}(r, s, b)=\sum_{i=0}^{\infty} c_{i} p_{i}(r, s,-1, b-1) .
$$

One gets recursively for the $K_{n}$ 's

$$
K_{n}(r, s, b)=\sum_{i=0}^{\infty} c_{i-n} p_{i}(r, s, n-1, n+b-1) .
$$

In (2.5) the $c_{i}$ 's $(i \geqq 0)$ are arbitrary constants; in the integrations (2.6), we have inserted the new arbitrary constants under the name of $c_{i}$ with negative values of the index $i$.

The last and hardest thing we have to do is to calculate (if possible) the constants $c_{i}(-\infty \leqq i \leqq \infty)$ [see (2.5) and (2.6)] and the signs $\mu_{n}$ [see (2.1)] in such a way that the remaining Yang equation (1.3) will also be satisfied. For the moment the only solutions we have discovered depend on a real parameter $\lambda$ and can be written as

$$
\begin{aligned}
& c_{i}=0 \quad \text { if } \quad i \geqq b, \\
& c_{i}=\lambda^{i} c_{0}(b) \text { if } i<b \text {. }
\end{aligned}
$$

With these coefficients the formal series are polynomials, let us call them $K_{n}(b)$ for simplicity, which obey the identities below:

\section{Linear Identities}

$$
\begin{gathered}
K_{n}(b)=\left(\frac{1}{\lambda}+s-r\right) K_{n-1}(b)+r s K_{n-2}(b) \\
\sqrt{|\lambda|} K_{n}(b+1)=(1-\lambda(r+s)) K_{n}(b)+\frac{1}{\sqrt{|\lambda|}} \lambda s K_{n}(b-1) .
\end{gathered}
$$

Quadratic Identity

$$
\left(K_{n}(b)\right)^{2}-K_{n+1}(b) K_{n-1}(b)=\mu_{n}(b) r^{a_{n}} S^{b_{n}},
$$

provided

$$
\mu_{n}(b)=(-1)^{n-1}(-\lambda)^{b-2}\left(c_{0}\right)^{2} .
$$

We see immediately that the hemiticity relation $(1.5 b)$ fixes the values of the normalisation factor $c_{0}(b)$ in $(2.7)$; it must be equal to

$$
c_{0}(b)=|\lambda|^{\frac{2-b}{2}}
$$


The proofs of the relations (2.8)-(2.11), again with the proof that they imply Eq. (1.3), can be found in [8].

Thus the formulae (2.1)-(2.12) provide a family of self-dual fields indexed by two discrete parameters ( $n$ and $b$ ) and by a scale factor $\lambda$. The hermiticity of each solution is easily analysed knowing (1.5) and (2.1) and the signs $\mu_{n}(b)$ obtained from (2.11) and (2.12):

$$
\mu_{n}(b)=(-1)^{n-1}(-\varepsilon)^{b} \quad(\varepsilon=\operatorname{sgn}(\lambda))
$$

\section{Finite Action Solutions}

Most of the solutions constructed in the preceding section are of no physical interest because they have infinite action. However taking $n=0$ and $\varepsilon=-1$, one obtains a subfamily (indexed by $b$ ) of solutions with finite action.

Let us write the relevant solutions transformed by (1.6); using (2.10) one obtains

$$
(f, e, g)(b)=\frac{1}{K_{1}}\left(K_{0}, \bar{Y}^{-1} \bar{Z}^{b-1},-Y^{-1} Z^{b-1}\right) .
$$

The new $f$ defined in (3.1) are strictly negative and finite functions when restricted to euclidean space-time (i.e., $r \geqq 0, s \geqq 0$ ). Here we write in general the dominant terms of $K_{0}$ and $K_{1}$ near the origin together with their asymptotic behaviour; the other terms can easily be derived from (A.2) and (2.6)

$$
\begin{aligned}
& K_{0}(b)=|\lambda|^{\frac{2-b}{2}}\left(1+|\lambda|(s+(b-1) r)+\ldots+|\lambda|^{b-1}(r+s)^{b-1}\right), \\
& K_{1}(b)=-|\lambda|^{\frac{2-b}{2}}\left(1+|\lambda| b r+\ldots+|\lambda|^{b-1} r(r+s)^{b-2}\right) .
\end{aligned}
$$

Knowing (3.1), one can explicitly calculate the associated gauge quantities, particularly the action density $\mathscr{A}$ (see [8, formula (3.50)]). After a lengthy calculation, we have verified that the action density of the solutions (3.1) is finite and smooth everywhere over space-time; it depends only on $K_{0}$ through the formula

$$
\mathscr{A}(b)=-\square \square \ln K_{0}(b)
$$

For $b=1$, this function is identically zero, for $b=2,3$ it is respectively equal to

$$
\begin{gathered}
\mathscr{A}(2)=\frac{6|\lambda|^{2}}{K^{4}}, \quad K=1+|\lambda|(r+s), \\
\mathscr{A}(3)=\frac{2|\lambda|^{2}}{K^{4}}\left(K^{2}+24|\lambda| s K+27|\lambda|^{2} s^{2}\right), \quad K=1+|\lambda|(s+2 r)+|\lambda|^{2}(s+r)^{2} .
\end{gathered}
$$

The function (3.5) is invariant under $\mathrm{SO}(4)$ rotations, it admits a unique maximum at the origin, and decreases monotonically in all directions from the origin. The function (3.6) has neither local maximum nor minimum on euclidean space-time; the absolute maximum on this domain occurs at the points with $r=0$, $s \simeq 0.3$; on the $s=0$ axis, the function decreases monotonically. 
For larger values of $b$, we do not have the explicit dependence of $\mathscr{A}$ on $r$ and $s$ but we have verified numerically that the function $\mathscr{A}$ has the same shape as (3.6).

Table 1. In the Table, we give approximate values of $\mathscr{A}_{\max }$ together with the coordinates $s_{\max }$ for the first few values of $b ; r_{\max }=0$

\begin{tabular}{lll}
\hline$b$ & $\begin{array}{l}S_{\text {max }} \text { in } \\
\text { unit of }|\lambda|^{-1}\end{array}$ & $\begin{array}{l}\mathscr{A}_{\text {max }} \text { in } \\
\text { unit of }|\lambda|^{2}\end{array}$ \\
\hline 2 & 0.0 & 6.0 \\
3 & 0.3 & 7.7 \\
4 & 0.55 & 16.7 \\
5 & 0.68 & 34.6 \\
6 & 0.77 & 65.7 \\
\hline
\end{tabular}

The formula (3.4) together with the asymptotic behaviour of $K_{0}$ [see (3.2)] allows us to calculate the action as a function of $b$. Using Stokes' theorem, one obtains $A=16 \pi^{2}(b-1)$. Remembering that our solutions are self-dual, we also have the topological number $T$ of our solutions; it is equal to

$$
T=\frac{A}{16 \pi^{2}}=b-1 \text {. }
$$

Thus the family (3.1) contains solutions with arbitrary values for the topological number. For $b=1$, it is a pure gauge, for $b=2$, it is the famous instanton [3]; for larger values of $b$ our solutions are interesting in that they are different from the $k$-instanton of 'tHooft. Indeed these last admit an action density with $k$ separated local maxima, precisely attained at the different positions of the instantons.

\section{Conclusions}

In conclusion let us note several common points between our solutions (we will call them multi-instantons) and the recently discovered multi-monopoles in $[4,5]$ :

(i) Both solutions describe 5-parameters objects ( 4 positions +1 scale factor) located around a single point in space.

(ii) Topologically, multi-monopoles (respectively multi-instantons) behave as several single monopoles (respectively instantons) located at finite distance from the origin. They are characterised by an integer: the magnetic flux (respectively the topological number).

(iii) The multi-object does not have the same symmetry as the object, i.e. the multi-monopole is not spherically symmetric under $\mathrm{SO}(3)$ (as is the monopole) and the multi-instanton has not the full symmetry under $\mathrm{SO}(4)$ that the instanton does.

(iv) At least for the first few values of $b$, the energy density of the multimonopole (plotted against $s$ and $x_{3}$ ) and the action density of the multi-instanton (plotted against $r$ and $s$ ) have almost the same shape (see figures in [5]), i.e. with the absolute maximum delocalised from the origin. As a consequence the energy and action densities are maximal on a circle in space (space-time). 
For all these reasons, we think that the solutions presented in (3.1) describe particular cases of several instantons superposed at the same point and, consequently, in interaction with each other. The existence of such solutions was already announced in [9] but the authors did not give any explicit form in their paper.

\section{Appendix}

The differential equation $\left[r \partial_{r r}+s \partial_{s s}-a \partial_{r}-b \partial_{s}\right] P=0$, where $a$ and $b$ are constants, admits among its solutions homogeneous polynomials of arbitrary degree $d$; let us call them $p_{d}(r, s, a, b)$; these can be written

$$
p_{d}(r, s, a, b)=\frac{1}{d !} \sum_{k=0}^{d} A_{d, k}(a, b) r^{k_{s} d-k},
$$

with the coefficients $A_{d, k}(a, b)$ given, up to a multiplicative constant, by ( $\Gamma$ denotes the usual Euler function)

$$
A_{d, k}(a, b)=(-1)^{k} \mathscr{C}_{d}^{k} \frac{\Gamma(a-k+1)}{\Gamma(a-d+1)} \cdot \frac{\Gamma(b-d+k+1)}{\Gamma(b-d+1)} .
$$

Then for $K_{0}$ in (2.5), we have chosen a general linear combination of the polynomials (A.1) together with the notation [see (2.4)]

$$
K_{m}\left(r, s, a_{n}, b_{n}\right) \equiv K_{n}(r, s, b) .
$$

Acknowledgements. I am grateful to Jean Nuyts for many helpful discussions and to Alex Martin for patiently reading the manuscript.

\section{References}

1. Atiyah, M.F., Hitchin, N.J., Drinfeld, V.G., Manin, Yu.I. : Construction of instantons. Phys. Lett. 65 A, 185 (1978)

2. Jackiw, R., Nohl, C., Rebbi, C.: Conformal properties of pseudoparticle configurations. Phys. Rev. D 15, $1642(1977)$

3. Belavin, A., Polyakov, A., Schwartz, A., Tyupkin, Yu.: Pseudoparticle solutions of the Yang-Mills equations. Phys. Lett. 59B, 85 (1975)

4. Prasad, M.K.: Yang-Mills-Higgs monopole solutions of arbitrary topological charge. Commun. Math. Phys. 80, 137 (1981)

5. Forgacs, P., Horwath, Z., Palla, L.: Generating monopoles of arbitrary charge by Bäcklund transformations. Phys. Lett. 102B, 131 (1981)

6. Yang, C.N. : Condition of self-duality for SU(2) gauge fields on Euclidean four-dimensional space. Phys. Rev. Lett. 38, 1377 (1977)

7. Brihaye, Y., Fairlie, D.B., Nuyts, J., Yates, R.G.: Properties of the self dual equations for an SU $(n)$ gauge theory. J. Math. Phys. 19, 2528 (1978)

8. Brihaye, Y.: Théorie de jauge. Quelques solutions classiques. Thèse de doctorat, Mons (1981)

9. Christ, N.E., Weinberg, E.J., Stanton, N.K. : General self-dual Yang-Mills solutions. Phys. Rev. D 18, 2013 (1978)

Communicated by R. Stora

Received September 2, 1981; in revised form December 10, 1981 